\title{
Broadband diamagnetism in anisotropic metamaterials
}

\author{
Mikhail Lapine, ${ }^{1,}$ Anastasia K. Krylova, ${ }^{2}$ Pavel A. Belov, ${ }^{2}$ Chris G. Poulton, ${ }^{3}$ Ross C. McPhedran, ${ }^{1}$ and Yuri S. Kivshar ${ }^{2,4}$ \\ ${ }^{1}$ CUDOS, School of Physics, University of Sydney, New South Wales 2006, Australia \\ ${ }^{2}$ National Research University of Information Technologies, Mechanics and Optics (ITMO), St. Petersburg 197101, Russia \\ ${ }^{3}$ CUDOS, School of Mathematical Sciences, University of Technology Sydney, New South Wales 2007, Australia \\ ${ }^{4}$ Nonlinear Physics Centre and CUDOS @ ANU, Australian National University, Australian Capital Territory 0200, Australia
}

(Received 27 August 2012; revised manuscript received 7 November 2012; published 11 January 2013)

\begin{abstract}
We discuss the strategy for achieving the values of the effective magnetic permeability much smaller than unity by employing an appropriate arrangement of metamaterial elements ("meta-atoms"). We demonstrate that strong diamagnetism over a very wide frequency range can be realized in metamaterials by employing nonresonant elements with deeply subwavelength dimensions. We analyze the effect of the lattice parameters on the diamagnetic response and find that selecting an appropriate lattice type is crucial for optimal performance. Finally, we discuss the optimal characteristics required to obtain the lowest possible values of magnetic permeability and point out an efficient tuning possibility.
\end{abstract}

DOI: 10.1103/PhysRevB.87.024408

PACS number(s): 81.05.Xj, 75.20.-g, 78.67.Pt

\section{INTRODUCTION}

Metamaterials have provided a wide playground for theoretical and applied electromagnetics, material science, and engineering for at least a decade, yielding a number of interesting designs and a range of novel phenomena and applications. Typically, ${ }^{1}$ the research on metamaterials is focused on negative refraction or other uses of negative effective parameters of metamaterials, which would be therefore based on resonant elements with a narrow-band response. The concept of metamaterials is, however, much broader, offering more flexibility and applications than assumed within the mainstream. ${ }^{2}$ This relies on a strong mutual interaction in metamaterials made possible by dense packing of their elements ("meta-atoms"). ${ }^{3,4}$ The consequence is a significant shift of the resonance frequency, ${ }^{3}$ offering convenient opportunities for tuning ${ }^{5}$ and nonlinear effects. ${ }^{6}$

However, mutual interaction is not only useful for affecting the resonance. As we show below, it is also helpful for tailoring the collective response of metamaterials' elements to gain unusual magnetic properties, for example, creating artificial diamagnetism with quite low effective permeability over a wide frequency range.

Artificial diamagnetics have been well known in engineering from early times, ${ }^{7}$ but normally it has been assumed that their effective permeability is not much smaller than unity. More recently, interest in this topic was revitalized in the context of metamaterials, ${ }^{8-10}$ with a suggestion to produce strong diamagnetism at vanishing frequency using a lattice of densely packed superconducting cubes or plates, ${ }^{8}$ and experimental approaches to measure such properties. ${ }^{11}$ A diamagnetic response of closed conducting rings was also recently revisited for a metamaterial built up with square split resonators or closed square loops. ${ }^{12}$ Unfortunately, the selected parameters were not optimal so the estimates in Ref. 12 did not predict particularly small permeability values, with the lowest suggested value being about 0.5 .

In this paper, we provide a comprehensive analysis of the diamagnetic properties available in dense anisotropic arrays of closed rings, offering a more refined treatment of the lattice effects (Sec. II). We discuss the optimal design parameters for implementations of such metamaterials and reveal that very low effective magnetic permeability (below 0.05) can be achieved in such structures (Sec. III). For certain parameters, the desired diamagnetic response easily spans a few orders of magnitude in frequency, as discussed in Sec. III F.

\section{EFFECTIVE PERMEABILITY IN NONRESONANT ARRAYS}

Our theoretical treatment is applicable when the size of one element as well as lattice constants are much smaller than the free-space wavelength, that is, in the quasistatic limit. Contrary to the case of resonant elements, where special efforts are required to provide a reliable description, ${ }^{13}$ here the size of the elements can be easily brought into the necessary range without compromising the performance. The upper frequency limit for our analysis can be taken such that the free space wavelength is still sufficiently in excess of the ring circumference, so the applicable range of angular frequencies is well below the characteristic threshold $\omega_{0}=c / 2 r, c$ being the light velocity and $r$ the radius of the rings.

For convenience in the equations, we take all the geometrical parameters normalized to $r$, so they can be written as dimensionless quantities. We assume all the rings to be arranged with identical orientation (all having parallel axes) with the lattice constants $a r$ in the ring planes and $b r$ along their axes (Fig. 1). In the plane of the rings, they can have tetragonal or hexagonal lattices, and the adjacent layers can be also shifted by ar $/ 2$, which provides a significant effect on the array properties ${ }^{3}$; as we show below, the lattice type is crucial for optimizing diamagnetic response. The density of the rings is then $n=a^{-2} b^{-1} r^{-3}$ for the tetragonal lattice and $n=2 / \sqrt{3} \cdot a^{-2} b^{-1} r^{-3}$ for the hexagonal lattice. For the sake of uniform notation, we introduce a geometrical factor $\gamma$ which equals 1 for tetragonal and $2 / \sqrt{3}$ for hexagonal lattices, so now we can generally write $n=\gamma \cdot a^{-2} b^{-1} r^{-3}$. For the sake of analytical simplicity, we assume the rings to have toroidal shape and introduce a dimensionless parameter $w$, which is the ratio of the radius of the wire which is used to make the rings, to the ring radius $r$; that is, the wire radius is $w r$. Geometrically, it is clear that $w<1, a>2(1+w)$, and $b>2 w$, but for the 


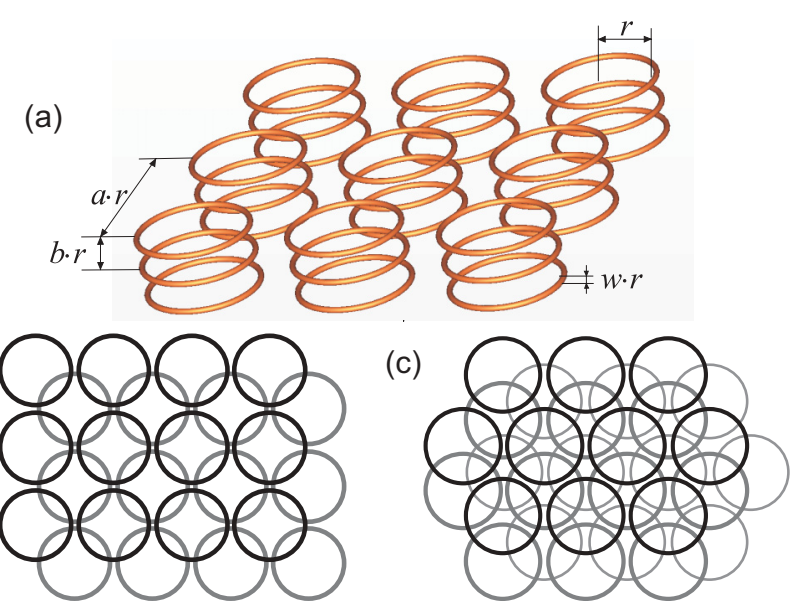

FIG. 1. (Color online) Various types of lattice arrangements. (a) General view of the anisotropic lattice (simple tetragonal arrangement in the plane) and possible modifications (top view). (b) Tetragonal lattice with each layer displaced by one half of the lattice constant. (c) Hexagonal lattice with each layer displaced by one half of the lattice constant.

analytical theory below to be precise, $w \ll 1$ is implied and $a$ and $b$ should be significantly larger than the above geometrical limits, to minimize the effect of capacitive coupling.

For dense arrays of conducting rings, their mutual interaction is particularly important and cannot be reduced to that with the nearest neighbors. A rigorous approach to determine quasistatic effective permeability in such a strongly coupled system was presented in Ref. 3 and we only outline the key steps here. Clearly, the macroscopic permeability can be calculated through spatial averaging of the local microscopic magnetic field and evaluation of the average magnetization, to be performed in a physically infinitesimal volume being much smaller than the wavelength and encompassing a sufficiently large number of unit cells. ${ }^{14,15}$ In the quasistatic limit, it can be assumed that the external field is uniform within that volume and therefore all the unit cells are identically excited with an external field $H_{0}$, inducing current $I$ in each ring. The averaging can be thus performed over one unit cell, and the average magnetization $M$ is determined by the magnetic moment $\pi r^{2} I$ of one ring divided by the unit cell volume: $M=n \pi r^{2} I$. However, the averaging procedure is less trivial than in conventional materials, because the specific shape of individual elements and their dense arrangement do not allow us to use the dipole approximation to calculate the local field and mutual effects. Instead, explicit fields of the individual currents have to be averaged, which can be done analytically, ${ }^{3}$ permitting us to express the permeability through the external (applied) magnetic field and induced magnetization as

$$
\mu=\left(H_{0}+\frac{2}{3} M\right) /\left(H_{0}-\frac{1}{3} M\right),
$$

which is valid for unbounded media or sufficiently large spherical samples.

In the quasistatic limit, a closed ring can be described as an effective contour characterized with resistance and inductance; however, the skin effect may become significant while the quasistatic consideration is still valid. For a consistent evaluation in the entire frequency range, we have to apply a general form of the impedance, ${ }^{15}$

$$
Z=-\mathrm{i} \omega L_{\mathrm{e}}+R \frac{\zeta}{2} \frac{J_{0}(\zeta)}{J_{1}(\zeta)}
$$

where $L_{\mathrm{e}}=\mu_{0} r(\ln (8 / w)-2)$ is the external contribution to the self-inductance, $R=2 \rho / r w^{2}$ is the static resistance, and $\zeta=(1+\mathrm{i}) r w / \delta$, is the argument to the zero $\left(J_{0}\right)$ and first $\left(J_{1}\right)$ order Bessel functions of the first kind $\left(\delta=\sqrt{2 \rho / \mu_{0} \omega}\right.$ is the skin depth).

Then the current $I$, excited in each ring, is determined by solving the corresponding Kirchhoff's equation,

$$
I=\frac{\mathrm{i} \omega \pi r^{2} \mu_{0} H_{0}}{\left(Z-\mathrm{i} \omega \sum_{n \neq n^{\prime}} L_{n n^{\prime}}\right)},
$$

where the mutual interactions are manifested by the summation over the mutual inductances between the rings. ${ }^{3}$ The result of this summation can be conveniently expressed as $\sum_{n \neq n^{\prime}} L_{n n^{\prime}}=\mu_{0} r \Sigma$, where $\Sigma$ is dimensionless, so that we can define the total inductance as $L_{\Sigma}=L_{\mathrm{e}}+\mu_{0} r \Sigma$. As explained in Ref. 3, the summation should be limited to the interior of the sphere which corresponds to the macroscopic averaging. In practice, it is sufficient to perform this summation within a radius of several lattice constants, which corresponds to a characteristic length of response formation. ${ }^{3,14}$ For dense arrays, contribution of the mutual inductances can be much larger than the self-inductance, providing a dominant part of the impedance.

It is then straightforward to obtain the effective permeability of this metamaterial as

$$
\mu=1-\left[\frac{b a^{2}}{\gamma \pi^{2}}\left(\frac{L_{\Sigma}}{\mu_{0} r}-\frac{1}{\zeta} \frac{J_{0}(\zeta)}{J_{1}(\zeta)}\right)+\frac{1}{3}\right]^{-1},
$$

where, we recall, $\zeta=(\mathrm{i}+1) w r \sqrt{\mu_{0} \omega / 2 \rho}$.

\section{DIAMAGNETIC PROPERTIES}

Analyzing expression (4), we can conclude that in the limit of low frequency its real part tends to unity and its imaginary part to zero, as expected for a nonmagnetic material. In the opposite limit of high frequency, more specifically, when $\operatorname{Im} Z \gg \operatorname{Re} Z$ (provided that the corresponding frequency is within the quasistatic limitations), the real part tends to the value

$$
\bar{\mu}^{\prime}=1-\left[\frac{b a^{2}}{\gamma \pi^{2}}\left(\Sigma+\ln \frac{8}{w}-2\right)+\frac{1}{3}\right]^{-1},
$$

which is below unity. We should emphasize that the latter expression is independent of the absolute scale of the problem, which only manifests itself in the level of dissipation (see Sec. IIIE) and in the diamagnetic transition frequency (see Sec. III F).

A few examples showing the general appearance of the permeability (4) are provided in Fig. 2. We start with a tetragonal lattice with $a=2.5$ and $b=0.5$ (this roughly corresponds to an arrangement considered in Ref. 12) and the normalized wire radius $w=0.1$; the diamagnetic response extends for a few orders of magnitude in frequency and reaches an effective permeability of about 0.5 (see the dotted curves). 


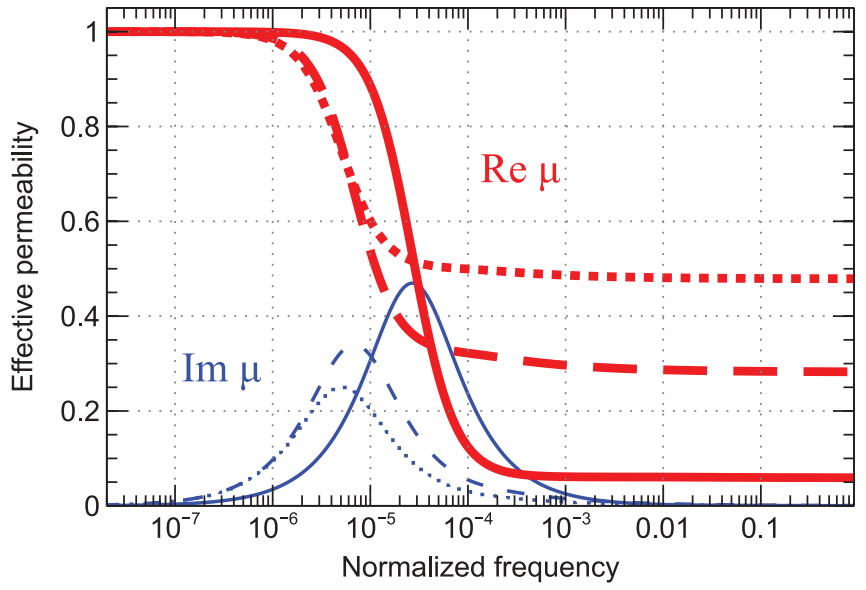

FIG. 2. (Color online) Effective permeability for different lattices: normal tetragonal lattice with $a=2.5, b=0.5, w=0.1$ (dotted curves), shifted tetragonal lattice with the same parameters (dashed curves), and shifted hexagonal lattice with $a=2.04, b=$ $0.02, w=0.009$ (solid curves). The real part is shown with red thick lines and the imaginary part with blue thin lines. Frequency is normalized to the threshold value $\omega_{0}$. Radius of the ring $r=500 \mu \mathrm{m}$.

However, a mere transition to a shifted tetragonal lattice with the same parameters (dashed curves) provides a remarkable improvement with the permeability going below 0.3 . Further enhancement is demonstrated with a shifted hexagonal lattice with a more dense packing, $a=2.02, b=0.02, w=0.009$, where the permeability plummets to 0.06 , with just a slightly smaller frequency range. Note that, while the plots extend up to the threshold frequency $\omega_{0}$, the actual physical limit for this theory to be reliable is at about $0.1 \omega_{0}$. In these examples, the ring radius was assumed to be $500 \mu \mathrm{m}$, but as we explain in detail in Sec. III F, the problem is very well scalable.

The plots clearly show the predicted behavior, with the real part of $\mu$ transiting from unity in the low-frequency limit to diamagnetic values at higher frequencies, and the imaginary part having its maximum in the area of transition. More specifically, the maximum of the imaginary part corresponds to the frequency $\tilde{\omega}$, where $\operatorname{Re} Z \sim \operatorname{Im} Z$; for certain parameters, $\operatorname{Im} \mu$ at its maximum may exceed the corresponding $\operatorname{Re} \mu$ part.

\section{A. Limitations of the theoretical approach}

Before we proceed to a detailed parametric analysis, we would like to indicate the limitations of the theory developed above. The standard restrictions arising from a quasistatic consideration, as discussed in Sec. II, are not so crucial for our results because the system is nonresonant and the transition to the diamagnetic regime typically occurs well below the quasistatic validity threshold; these details are discussed in Sec. III F.

More relevant is the precision in the calculations of the lattice sum $\Sigma$, which implies the mutual inductance be evaluated within a filament current approximation. This, however, may be inaccurate for the nearest neighbors in case the wire radius is not much smaller than the ring radius and, at the same time, the lattice constants are not much larger than the wire diameter. In such a case, current redistribution would affect the mutual inductance.
To provide a quantitative evaluation of possible discrepancy, we note the following: Given the symmetry of the problem, it is clear that the most extreme current distribution in this system would correspond to, effectively, two linear circular currents flowing along the opposite points of the wire cross section, as displaced from the wire center in $z$ direction. While we do not argue this is the actual distribution, this case represents a maximum possible deviation from a filament current approximation, thus being suitable for an upper estimate.

For such an extreme case, we can evaluate the effective mutual inductance in a way, analogous to the mutual inductance between the flat rings of finite width. ${ }^{16}$ It was shown that, despite a nontrivial current profile in the ring cross section, for inductance calculations, each ring may be represented with two filament currents positioned within the cross section at a certain distance from each other, and then the effective mutual inductance can be calculated as the average between the four emerging mutual inductances [see Eq. (6) and the text on p. 1134 of the Ref. 16]. This method provides a very good match to the exact numerical calculation based on full-wave simulations.

Adopting this approach, we have checked how much the mutual inductance calculated for the extreme configuration described above differs from that between single filaments. As expected, the discrepancy was only found to be remarkable when $b$ is close to $2 w$ and $w$ is not much smaller than unity. In turn, this is reflected in the overall calculations of the lattice sum $\Sigma$ to a smaller extent, because only the nearest neighbors are significantly affected. We note that the difference was also visible for shifted lattices, although to a much smaller degree; for sufficiently thin wire $(w<0.01)$ the discrepancy is small even with $b$ quite close to $2 w$. In the analysis below, we take this concern into account and provide the corresponding comparison where relevant.

\section{B. General remarks on parametric features}

Having achieved the desired result that the permeability (5) is remarkably diamagnetic in a wide frequency range, we now proceed to a more detailed parametric analysis and discussion of the optimal configuration.

We can see that the lattice constants and type play a crucial role in determining the minimum achievable value (5). First of all, we note that a hexagonal lattice produces a stronger diamagnetism through a larger density and slightly smaller $\Sigma$; and then $\Sigma$ can be minimized even further by choosing a shifted lattice.

Then, a decrease in $a$ and $b$ generally works towards minimizing $\bar{\mu}$, both through an increasing density. For minimizing $a>2$ there is no such freedom, but $b$ can be made quite small. The appearance of Eq. (5) may give an impression that $\bar{\mu}$ can be made arbitrarily small or even negative in the limit $b \rightarrow 0$. This, however, is not true because the decrease of $b$ is compensated by the corresponding increase in $\Sigma$. On the other hand, $\bar{\mu}$ decreases with a growth of $w$, which acts against the recommendation of making lattice constants as small as possible.

The final balance can be numerically revealed from Eq. (5) by assuming that the lattice constants are put to minimal possible values for a given $w$. To obtain the asymptotic 


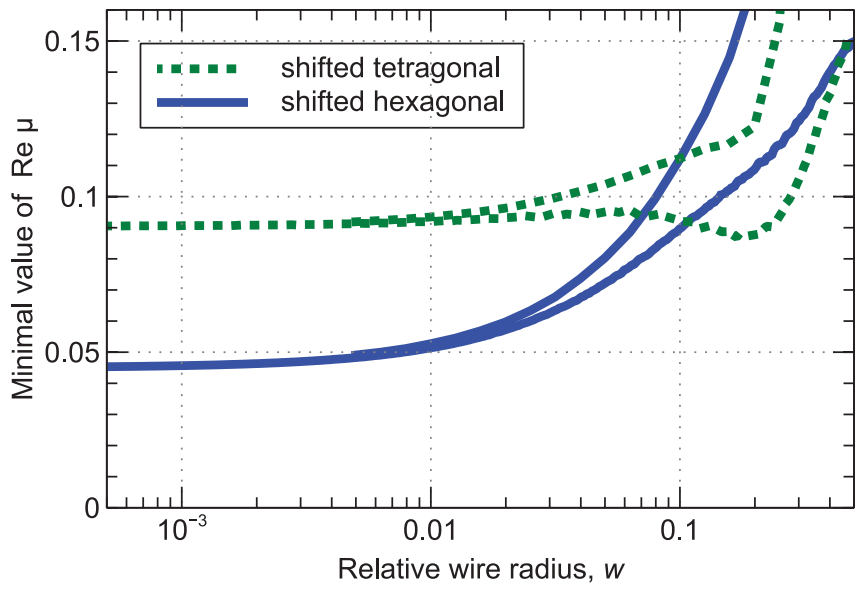

FIG. 3. (Color online) Asymptotic limits for the effective permeability which can be achieved in shifted tetragonal lattice (dashed green line) or hexagonal lattice (solid blue line), depending on $w$ with appropriately varied $a$ and $b$. In each case, the lower curves correspond to filament current approximation with the lattice parameters varied as $b=2 w$ and $a=2+b$, and the upper curves correspond to an extreme current distribution (see the text) with $b=2 w /(1+w)$ and $a=2+b$. The actual values must lie between the two curves in each case.

limitations, a parametric analysis on $w$ can be performed taking formally $a=2+b$ and $b=2 w$. This, however, is increasingly inaccurate when $w \ll 1$, and we therefore also provide a calculation for the most extreme current redistribution (see Sec. III A for details), taking a small additional offset between the rings so that $b=2 w /(1+w)$ and $a=2+b$. Such calculations are reasonable for shifted lattices because the rings in the neighboring layers in a shifted lattice get close to each other orthogonally at a few points only (unlike those in nonshifted lattices, lying next to each other along the entire circumference).

The resulting dependence of $\bar{\mu}^{\prime}$ on $w$ for shifted lattices is shown in Fig. 3. We can see that diamagnetic values approaching 0.05 are likely to be achieved; however, there is no need to take $w$ much smaller than 0.01 because a sufficiently low magnitude of $\bar{\mu}^{\prime}$ is already achieved and very little progress can be made by changing to smaller values of $a, b$, and $w$. Note that for relatively large $w>0.1$, the discrepancy between the filament current model and maximal possible deviation is quite large even for shifted lattices, so in this range it would be necessary to rely on exact numerical calculations to assess the corresponding design reliably.

\section{Efficient tuning possibility}

Despite the explicit quadratic dependence on $a$ in Eq. (5), the actual behavior of $\bar{\mu}^{\prime}$ is not so trivial for shifted lattices, because of the strong variations in the lattice sum $\Sigma$. We illustrate this in Fig. 4, where in the case of the shifted hexagonal lattice it is not even monotonic, showing an additional local minimum at $a \approx 3.3$. The reason for this is an extremely strong initial decrease in $\Sigma$ values for shifted lattices when $a$ increases, occurring because of the negative contribution from the rings in the displaced layers, which, through shifting, effectively transit from coaxial to almost

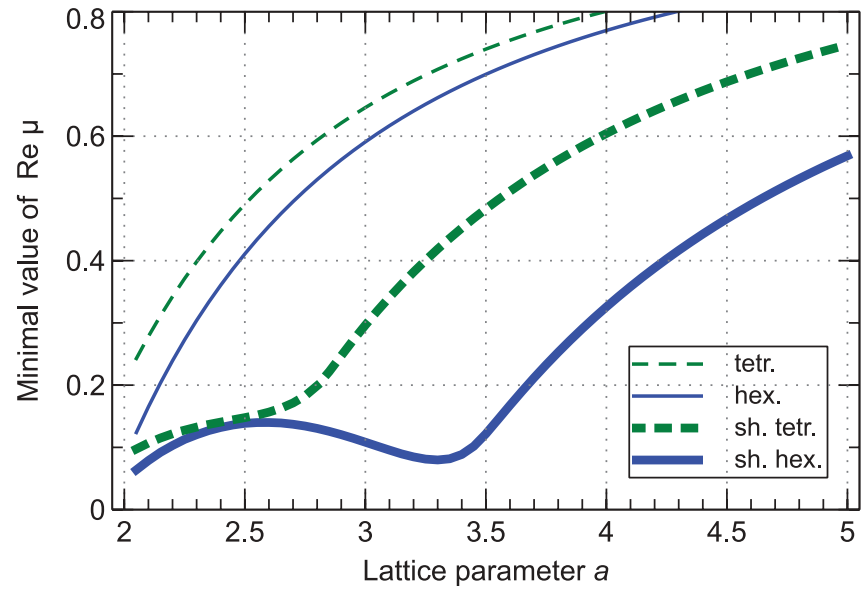

FIG. 4. (Color online) Minimal values of the effective permeability in shifted (thick lines) or nonshifted (thin lines) tetragonal (dashed green line) or hexagonal (solid blue line) lattices with various $a$. In these examples, $b=0.05$ and $w=0.02$.

coplanar configuration. Their contribution is most negative when the vertical overlap between the areas enclosed by the rings vanishes, which is only achieved when the rings are appropriately diluted in the plane so that in the shifted configuration the rings do not have overlapping neighbors in the adjacent layers.

Nonshifted lattices, on the contrary, feature a rapid monotonic increase with $a$. This behavior is very promising for implementing reconfigurable diamagnetism with the lattice tuning mechanism, ${ }^{5}$ whereby the lattice structure can be gradually controlled. Indeed, the difference between the permeability for a shifted and nonshifted lattice can be a factor of 8 , and for tuning applications it is most efficient to use hexagonal lattices with $a \approx 3.3$ and small $b$ (e.g., 0.05 ), where the permeability can be continuously tuned between 0.66 and 0.08 by shifting the lattice in a mechanically reconfigurable structure. In Fig. 5, we illustrate the efficiency of tuning by showing the ratio of the permeability between nonshifted and

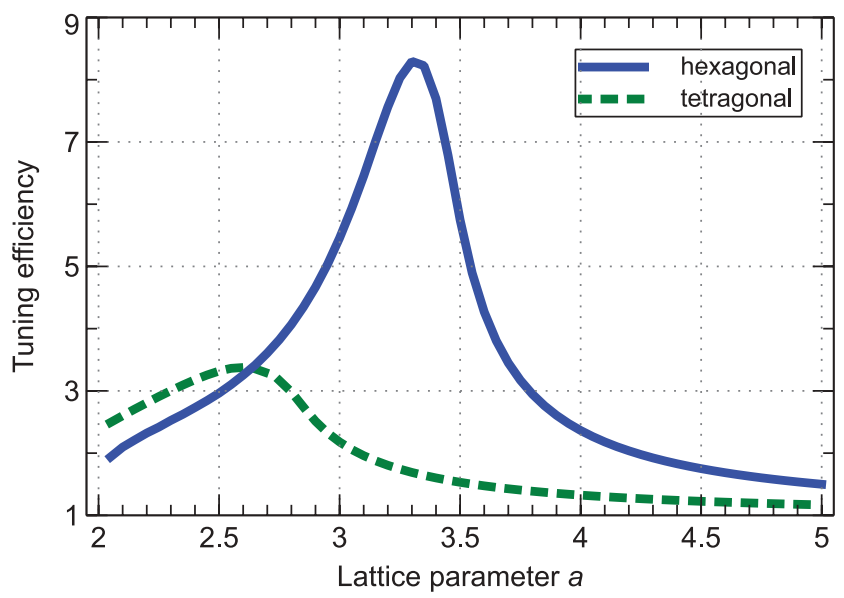

FIG. 5. (Color online) Tuning efficiency for hexagonal (solid blue line) and tetragonal (dashed green line) lattices, calculated as the ratio between the minimal values of the effective permeability in the nonshifted and entirely shifted structure. Shown for various $a$ at $b=0.05$ and $w=0.02$. 


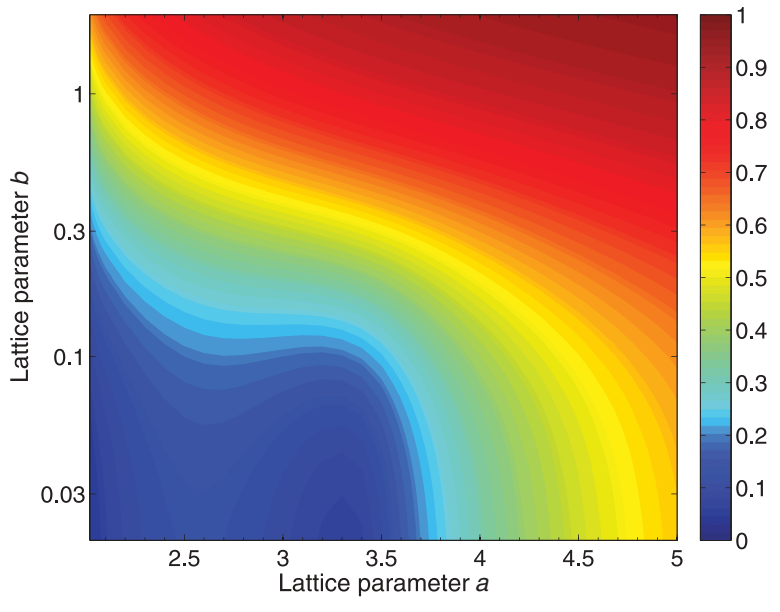

FIG. 6. (Color online) Minimal values of the effective permeability (as indicated by the color bar) in shifted hexagonal lattices with various $a$ and $b$ and fixed $w=0.01$; note the logarithmic scale on $b$ (vertical axis).

fully shifted lattices with various $a$, with a fixed $b=0.05$ and $w=0.02$. An important observation is that quite specific parameters are required for good tuning, otherwise the tuning range can be rather small. The hexagonal lattice is dramatically more efficient as compared with the tetragonal arrangement.

To provide a complete picture of the corresponding parametric dependence, we show a two-dimensional parametric plot (Fig. 6), where $\bar{\mu}^{\prime}$ is shown for a range of $a$ and $b$ parameters for a shifted hexagonal lattice, calculated with $w=0.01$ (which suits the minimal lattice parameters used for this figure). The plot shows that the overall dependence is not so trivial and provides a useful insight for future design.

\section{Minimal values of the permeability}

To comment on the limiting values of $\bar{\mu}^{\prime}$, we need to simplify Eq. (5) by using an approximate expression for $\Sigma$. By analyzing the numerically obtained values of $\Sigma$ for various lattices, we were able to conclude that the following functional form fits the numerical data with a very good accuracy and can be used when $b<1$,

$$
\left.\Sigma\right|_{a \rightarrow 2} \approx C+\frac{D}{b}+\ln (b),
$$

where the coefficients $C$ and $D$ depend on the lattice type and $a$ parameter (for example, for a shifted hexagonal lattice with $a \rightarrow 2, C \approx-1.95, D \approx 2.03$; for a shifted tetragonal lattice, $C \approx-2.4, D \approx 1.89$ ). Taking, formally, $b=2 w$ and $a=2(1+w)$, we obtain

$$
\lim _{w \rightarrow b / 2} \bar{\mu}^{\prime}=1-\left(\frac{(2+b)^{2}}{\gamma \pi^{2}}[D+b(C+\ln 16-2)]+\frac{1}{3}\right)^{-1},
$$

where we now can take the limit of $b \rightarrow 0$ :

$$
\lim _{w \rightarrow b / 2} \bar{\mu}^{\prime} \stackrel{b \rightarrow 0}{\longrightarrow} 1-\left(\frac{4 D}{\gamma \pi^{2}}+\frac{1}{3}\right)^{-1} .
$$

Clearly, this value must be larger than zero, so it is necessary that $D / \gamma>\pi^{2} / 6 \approx 1.65$; this requirement is fulfilled for all the lattices. Formally, for the $D$ and $\gamma$ values corresponding to the shifted hexagonal lattice, expression (7) would give an ultimate limit at $\approx 0.034$; however, this is unlikely to be within a practical reach, keeping in mind the limitations and approximations assumed with the above theory.

\section{E. Dissipation in the diamagnetic range}

The trend of the dissipation is less trivial as the imaginary part of the permeability depends on frequency as well as on $w$ and $r$ in a complex way. For the optimal parameters discussed above, it is possible to express some of the asymptotic dependencies in a simple form, where we introduce a dimensionless frequency offset $v=\omega / \omega_{0}$. For the range where skin effect is essential, we obtain

$$
\left.\operatorname{Im} \mu\right|_{w \gg \delta / r}=\frac{\frac{\gamma \pi^{2}}{4(1+w)^{2}} \frac{\delta}{r}}{\left(D+\frac{\gamma \pi^{2}}{12(1+w)^{2}}+2 w(\ln 16-2+C)\right)^{2}}
$$

and for $w \ll 1$ it can be further simplified as

$$
\left.\operatorname{Im} \mu\right|_{\delta / r \ll w \ll 1}=\frac{\gamma \pi^{2} \sqrt{\rho /\left(\eta_{0} r v\right)}}{2\left(D+\gamma \pi^{2} / 12\right)^{2}}=\frac{\Xi \sqrt{\rho / \eta_{0}}}{2 \sqrt{r \nu}},
$$

where $\eta_{0}=\sqrt{\mu_{0} / \varepsilon_{0}}$ and $\Xi=\frac{\gamma \pi^{2}}{\left(D+\gamma \pi^{2} / 12\right)^{2}}$ is a dimensionless coefficient that gathers all the quantities related to lattice geometry. For example, a shifted hexagonal lattice has $\Xi \approx 1.28$, and a shifted tetragonal one has $\Xi \approx 1.34$. Then, for the rings made of copper, $\sqrt{\rho / \eta_{0}} \approx 6.3 \times 10^{-6}\left[\mathrm{~m}^{0.5}\right]$, so the numerical factor in Eq. (9) is about $4 \times 10^{-6}\left[\mathrm{~m}^{0.5}\right]$. Expression (9) shows that in the range $\delta / r \ll w \ll 1$, dissipation increases with diminishing $r$, but is approximately independent of $w$.

For much smaller $w$, such that the skin effect does not come into play, another asymptotic dependence can be derived,

$$
\left.\operatorname{Im} \mu\right|_{w \ll \delta / r}=\frac{\left(\gamma \pi^{2} / 2 w\right)(\delta / r)^{2}}{\left(D+\gamma \pi^{2} / 12\right)^{2}}=\frac{2 \Xi\left(\rho / \eta_{0}\right)}{w r v},
$$

where, for the same examples as above, the total numerical coefficient is about $\sim 10^{-10} \mathrm{~m}$. This behavior features stronger dependence on the absolute scale and now also on $w$.

The above asymptotic expressions, however, are only suitable for rough estimates, and in the intermediate range the use of the complete expression (4) is necessary. In Fig. 7 we show the magnitudes of $\operatorname{Im}(\mu)$ calculated with (4) for a wide range of $w$ and $r$.

\section{F. Absolute scaling of the problem}

The presented analysis is quite general in terms of applicable frequencies, so the results hold as long as the description of the conductive rings in terms of the specified contour parameters is valid. However, with increasing frequency the resistance will generally increase, implying that the transition to diamagnetic behavior at $\tilde{\omega}$ will occur at a relatively larger frequency. In other words, when diminishing all the dimensions, $\tilde{\omega}$ increases faster than the threshold frequency $\omega_{0}$. This limits the range where artificial diamagnetism can be observed at high frequencies, and our estimates show that with frequency approaching the optical range (where the theory is not applicable for other reasons anyway) diamagnetism is not achieved with this method.

Indeed, the ratio $\omega_{0} / \tilde{\omega}$ is proportional to $r$, which in this sense reflects the absolute scale of the problem. For the optimal 


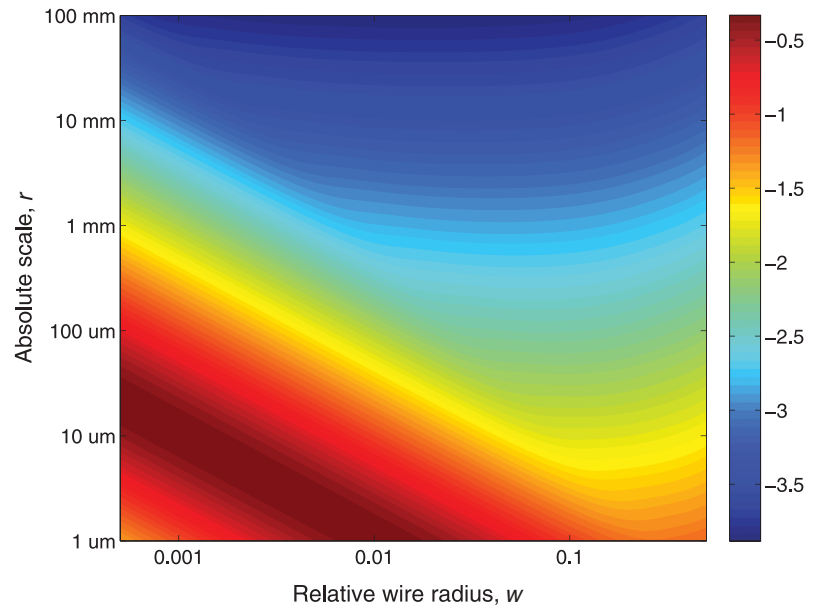

FIG. 7. (Color online) Decimal logarithm of the imaginary part of the effective permeability (the scale is shown with the color bar) observed at the relative frequency $v=0.01$ in a shifted hexagonal lattice with optimal parameters for various $w$ and $r$ values. All the quantities are shown in a logarithmic scale.

parameters discussed above $(a \rightarrow 2+2 w$ and $b \rightarrow 2 w)$ and $w$ well smaller than unity, it is also proportional to $w$ :

$$
\frac{\omega_{0}}{\tilde{\omega}} \approx \frac{\eta_{0}}{4 \rho} w r \text { for } w \ll 1,
$$

which is a useful equation for estimating quantitatively the available range of diamagnetic response.

As the characteristic frequency $\tilde{\omega}$ corresponds roughly to the maximum of the imaginary part of the effective permeability, at least an order of magnitude in frequency should be allowed before a practically relevant diamagnetic response is claimed. On the other hand, the frequency should

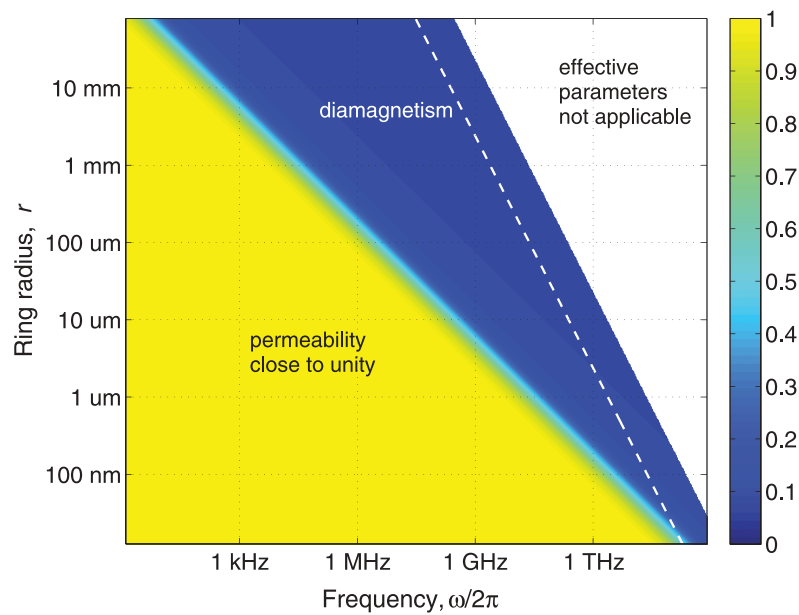

FIG. 8. (Color online) Real part of the effective permeability (the scale is shown with the color bar) observed at various frequencies between $1 \mathrm{~Hz}$ and $1 \mathrm{PHz}$ in metamaterials with different ring radii (from $10 \mathrm{~cm}$ to $10 \mathrm{~nm}$ ). The white dashed line indicates a reliability threshold taken as $\omega_{0} / 10$, so only the data to the left from this line can be used in practice; the abrupt cutoff to the white space on the right of this line is the calculation threshold at $\omega_{0}$. The axes are in logarithmic scale. not exceed about $0.1 \omega_{0}$ to avoid capacitive coupling and spatial dispersion. Therefore, the ratio (11) should be larger than 100 in order to have at least a small frequency range of practical diamagnetism. For a good conductor like copper, $\eta_{0} / \rho \approx 2.5 \times 10^{10} \mathrm{~m}^{-1}$, and therefore the absolute scaling limitation is $w r \gtrsim 1.6 \times 10^{-8}$, which requires $r$ to exceed about $200 \mathrm{~nm}$, which is anyway outside the frame of our analysis in terms of applicable frequency.

Thus, for example, with a ring radius of $1 \mu \mathrm{m}$ the range of practical diamagnetism falls between roughly 0.4 and $2.4 \mathrm{THz}$, while with a radius of $1 \mathrm{~mm}$ an impressive range from $400 \mathrm{kHz}$ to $2.4 \mathrm{GHz}$ can be used. An overall impression of the diamagnetic range is provided in Fig. 8, where the real part of the permeability is plotted for a huge range of frequencies and ring sizes (here, a shifted hexagonal lattice with $a=2.2$ and $b=0.2$ was used for calculations).

It is interesting to note that for good conductors, the diamagnetic transition at $\tilde{\omega}$ typically occurs before the skin effect becomes noticeably relevant, as the characteristic frequency of the latter, $\omega_{\mathrm{s}} \sim 2 \rho /\left(\mu_{0} w^{2} r_{0}^{2}\right)=\tilde{\omega} / w$, is always larger than $\tilde{\omega}$ for optimal parameters.

\section{CONCLUDING REMARKS}

We have shown that with an appropriate choice of the structural parameters in anisotropic lattices of closed rings, rather low effective permeability - down to at least 0.05 - can be reached. The best characteristics are obtained with the wire radius being as large as possible to fit the lattice geometry, and shifted lattices provide a remarkable improvement. To achieve the magnitudes below 0.1, both shifted tetragonal and shifted hexagonal lattices are suitable, and the required lattice constants can be about $a<2.2, b<0.05$ for tetragonal configurations and about $a<3.5, b<0.2$ for hexagonal configurations. For lower magnitudes, the shifted hexagonal lattice is the only choice, allowing us to obtain a permeability below 0.05 with $a<2.02$ and $b<0.02$.

These low values of magnetic permeability are expected in a very wide frequency range, easily spanning several decades in frequency, and are accompanied by quite small dissipation. The required geometry involves quite a small fraction of metal, making it useful for practical applications which require lowweight anisotropic diamagnetic response, for example, in the design of cloaking or levitating systems.

We have revealed the remarkable effect of the lattice type, particularly of a shift of the neighboring layers, that can be employed for making dynamically tunable diamagnetics with the structural reconfiguration method. ${ }^{5}$ Indeed, implementing that kind of tuning to our diamagnetic metamaterials would result in a remarkable tunability range, making it possible to change the magnetic permeability by a factor of 8 with a simple lattice shift.

Finally, we should note that our analysis has been carried out for circular elements in order to present the results in a simple analytical form. However, our results suggest that using those shapes which would provide a more dense packing, for example, a tetragonal array of shifted squares, may yield even smaller values of the permeability.

Achieving low permeability while keeping, at the same time, low weight of the structure, can be quite helpful to 
facilitate magnetic levitation, ${ }^{17}$ and the array of conductive rings considered in this work appears to be suitable for such use. Also, with the lowest nonresonant magnetic permeability considered so far, this analysis may pave a road towards the so-called MNZ (permeability near zero) materials. ${ }^{18}$ We therefore believe that the analysis above will bring a useful inspiration to future metamaterials research.

\section{ACKNOWLEDGMENTS}

The authors are grateful to Constantin R. Simovski, Lukas Jelinek, Stanislav I. Maslovski, and Maxim V. Gorkunov for the useful discussions. This work was supported by the Ministry of Education and Science of Russian Federation (Project 11.G34.31.0020) and by the Australian Research Council (Project No. DP110105484 and CUDOS Centre of Excellence CE110001018).
*Corresponding author: mlapine@physics.usyd.edu.au

${ }^{1}$ J. B. Pendry, New Sci. 209, ii (2011).

${ }^{2}$ M. Lapine and S. Tretyakov, IET Proc. Microwaves, Antennas Propag. 1, 3 (2007).

${ }^{3}$ M. Gorkunov, M. Lapine, E. Shamonina, and K. H. Ringhofer, Eur. Phys. J. B 28, 263 (2002).

${ }^{4}$ E. Shamonina, V. A. Kalinin, K. H. Ringhofer, and L. Solymar, J. Appl. Phys. 92, 6252 (2002).

${ }^{5}$ M. Lapine, M. Gorkunov, D. A. Powell, I. V. Shadrivov, R. Marqués, and Y. S. Kivshar, Appl. Phys. Lett. 95, 084105 (2009).

${ }^{6}$ M. Lapine, I. V. Shadrivov, D. A. Powell, and Y. S. Kivshar, Nat. Mater. 11, 30 (2012).

${ }^{7}$ S. A. Schelkunoff and H. T. Friis, Antennas Theory and Practice (Wiley, Nerw York, 1966).

${ }^{8}$ B. Wood and J. B. Pendry, J. Phys.: Condens. Matter 19, 076208 (2007).

${ }^{9}$ J. Shin, J.-T. Shen, and S. Fan, Phys. Rev. Lett. 102, 093903 (2009).
${ }^{10}$ C. Navau, D.-X. Chen, A. Sanchez, and N. Del-Valle, Appl. Phys. Lett. 94, 242501 (2009).

${ }^{11}$ F. Magnus, B. Wood, J. Moore, K. Morrison, G. Perkins, J. Fyson, M. C. K. Wiltshire, D. Caplin, L. F. Cohen, and J. B. Pendry, Nat. Mater. 7, 295 (2008).

${ }^{12}$ E. N. Economou, T. Koschny, and C. M. Soukoulis, Phys. Rev. B 77, 092401 (2008).

${ }^{13}$ C. R. Simovski, Opt. Spectrosc. 107, 726 (2009).

${ }^{14}$ M. I. Ryazanov, Condensed Matter Electrodynamics (Nauka, Moscow, 1984).

${ }^{15}$ L. D. Landau and E. M. Lifschitz, Electrodynamics of Continuous Media (Pergamon Press, Oxford, 1984).

${ }^{16}$ M. Lapine, L. Jelinek, R. Marqués, and M. J. Freire, IET Microwaves, Antennas Propag. 4, 1132 (2010).

${ }^{17}$ Y. Urzhumov, W. Chen, C. Bingham, W. Padilla, and D. R. Smith, Phys. Rev. B 85, 054430 (2012).

${ }^{18}$ N. Engheta, A. Alù, M. G. Silveirinha, A. Salandrino, and J. Li, Proceedings of the Mediterranean Electrotechnical Conference, 2006 (IEEE, New York, 2006), pp. 258-261. 